\title{
Cadaver skin allograft may improve mortality rate for burns involving over $30 \%$ of total body surface area: a propensity score analysis of data from four burn centers
}

\author{
Young Hwan Choi • Young Soon Cho 1 - Jong Ho Lee $\cdot$ Yanghwan Choi • \\ Soo Young Noh - Seungchoon Park - Changmin Sung • Jin Kyu Lim • \\ Jongdae Kim · Jae Jun Shin · Banseok Yang · Jiyun Jeong • Huan Chun • \\ Kwang Jo Kim
}

Received: 2 April 2018/Accepted: 25 July 2018/Published online: 31 July 2018

(C) Springer Nature B.V. 2018

\begin{abstract}
Cadaver skin is used for temporary wound covering, but there is insufficient evidence regarding its clinical usefulness in patients with major burns. We aimed to analyze the effect of cadaveric skin allograft on mortality rates in patients with burns involving $>30 \%$ of total body surface area (TBSA). Our study included 1282 patients with $>30 \%$ of TBSA burned admitted to four hospitals in Korea between June 1, 2008 and December 31, 2016. Of these, 698 patients underwent cadaver skin allograft (cadaver group), and 584 were treated with conventional treatment (noncadaver group). We corrected the differences between the two groups using propensity score matching, and generated 474 propensity score-matched pairs. Overall 90-day in-hospital mortality rate among all patients was $35.3 \%$ (453/1282). There was a significant difference in 90-day in-hospital mortality between the two groups for both unmatched [cadaver vs.
\end{abstract}

Y. H. Choi · J. H. Lee · Y. Choi · C. Sung

Department of Emergency Medicine, Bestian Hospital,

Seoul, Korea

Y. S. Cho $(\bowtie)$

Department of Emergency Medicine, Soonchunhyang University Bucheon Hospital, 1174, Jung-dong, Wonmigu, Bucheon-si, Gyeonggi-do 420-020, Korea e-mail: emer0717@nate.com

S. Y. Noh · S. Park · J. K. Lim · J. Kim ·

J. J. Shin · B. Yang · J. Jeong · H. Chun · K. J. Kim Department of General Surgery, Bestian Hospital, Seoul, Korea conventional, 31.7 vs. $39.7 \%$; difference, 8.0 ; $95 \%$ confidence interval (CI) 2.8-13.3] and propensitymatched groups ( 37.8 vs. $47.3 \%$; difference, $9.5 ; 95 \%$ CI 3.2-15.8). Logistic regression analyses showed a significant association between cadaver skin allograft and lower 90-day in-hospital mortality in the propensity-matched groups (odds ratio, 0.42; 95\% CI 0.29-0.62). Patients with major burns who underwent cadaver skin allograft had a lower mortality rate compared to those who did not. Cadaver skin allograft may improve the survival of patients with major burns, especially in the early phase of injury.

Keywords Allograft - Cadaver skin · Major burn

\section{Introduction}

Traditionally, patients with burns were treated with dressings and topical antimicrobial agents until separation of the eschar. The wound with granulation tissue formation was then covered with a graft after approximately 3-5 weeks. However, patients with major burns treated in the traditional manner are more likely to die from complications such as sepsis. In the early 1940s, it was recognized that removal of the burn eschar and immediate skin grafting were the most important steps in reducing mortality rate from major thermal injury (Cope et al. 1947). 
In 1970, Janžekovic (1970) treated patients with large burns using early tangential excision and covered the wound with grafts within 5 days of the burn, reducing mortality rates and hospital time. Thereafter, the usefulness of early excision has been widely reported in clinical trials by a number of surgeons, including Professor John Burke (Burke et al. 1974; Engrav et al. 1983; Herndon et al. 1989; Tompkins et al. 1988). Today, it is widely accepted that large burns should be excised quickly, usually within 5-7 days following injury, before infection can become established. Generally, early excision is performed within the first week or 10 days after injury, before spontaneous sloughing or invasive infection can occur (Subcommittee et al. 2016).

The ideal wound covering after early excision is autografting. However, in certain cases, autografting is not feasible, or unlikely to succeed; in these situations, the wound should be covered after early excision. A range of materials for temporary wound covering is available when grafting immediately after early excision is not possible.

Cadaver skin is one of the materials used for temporary wound covering. It reduces pain, decreases the loss of water and heat, and prepares the wound bed for grafting (Greenfield et al. 2001; Lawton and Dheansa 2008; Leon-Villapalos et al. 2010). Though clinical trials provided some data on cadaver skin grafting (Dhennin et al. 2002; Druecke et al. 2002; Fletcher et al. 2013; Khoo et al. 2010; Naoum et al. 2004; Vloemans et al. 2002), there is currently limited evidence on the clinical usefulness of cadaver skin in patients with major burns.

In this study, we analyzed the records of patients with burns covering over $30 \%$ of total body surface area (TBSA). The patients were treated in a similar manner over 8 years in four different hospitals. We aimed to examine the effect of cadaveric skin allografts on patient mortality rate.

\section{Methods}

Subjects

The design of this retrospective study was reviewed and approved by the institutional review board of Bestian Hospital (2017-08-001). We retrospectively analyzed the electronic medical records of patients admitted to four hospitals in Korea between June 1, 2008 and December 31, 2016. This study included patients with burns affecting over $30 \%$ of TBSA. Patients with sunburns, patients transferred to another hospital within 7 days of the burn, and patients with a diagnosis of death on arrival were excluded. All surgeons working in the four hospitals performed cadaveric skin allografts using similar surgical techniques. The common surgical protocol was as follows: cadaveric skin grafts were generally performed within 7 days of the injury and autografting of the wound with cadaveric skin was performed approximately 2 weeks later. We performed several autografts in succession until the wound area was small enough to be healed without autograft.

We categorized the patients according to whether they received cadaver skin allograft (cadaver group) or not (non-cadaver group). The following data were collected: demographic characteristics (age, sex), TBSA, full-thickness burns, inhalation burn, intensive care unit (ICU) admission, ventilator use, abbreviated burn severity index, and in-hospital mortality. The primary outcome measure for this study was inhospital mortality. All surgeons working in the four hospitals included in this study performed cadaveric skin allografts (cryopreserved) using similar surgical techniques. We used meshed skin allograft from 2008 to 2011 and unmeshed skin allograft from 2012.

\section{Statistical analysis}

The general characteristics were compared using independent $t$ test for continuous variables and Chi squared test for categorical variables. The propensity score was estimated using the logistic regression model for the cadaver group as a function of patient demographic and clinical characteristics. We included seven factors that were considered potentially clinically significant: age, sex, TBSA, full-thickness burn, inhalation burn, ICU admission, and ventilator use. Some of these variables significantly differed between the cadaver and non-cadaver groups. The score represented the predicted probability that a patient would be treated with cadaver skin allograft. We performed a one-to-one matching analysis between the cadaver and non-cadaver groups, using the nearest neighbor method with the closest propensity scores within 0.2 on a scale of $0-1$ (Fig. 1). Propensity matching is an analog to the process of randomization 
Fig. 1 Patient flow diagram

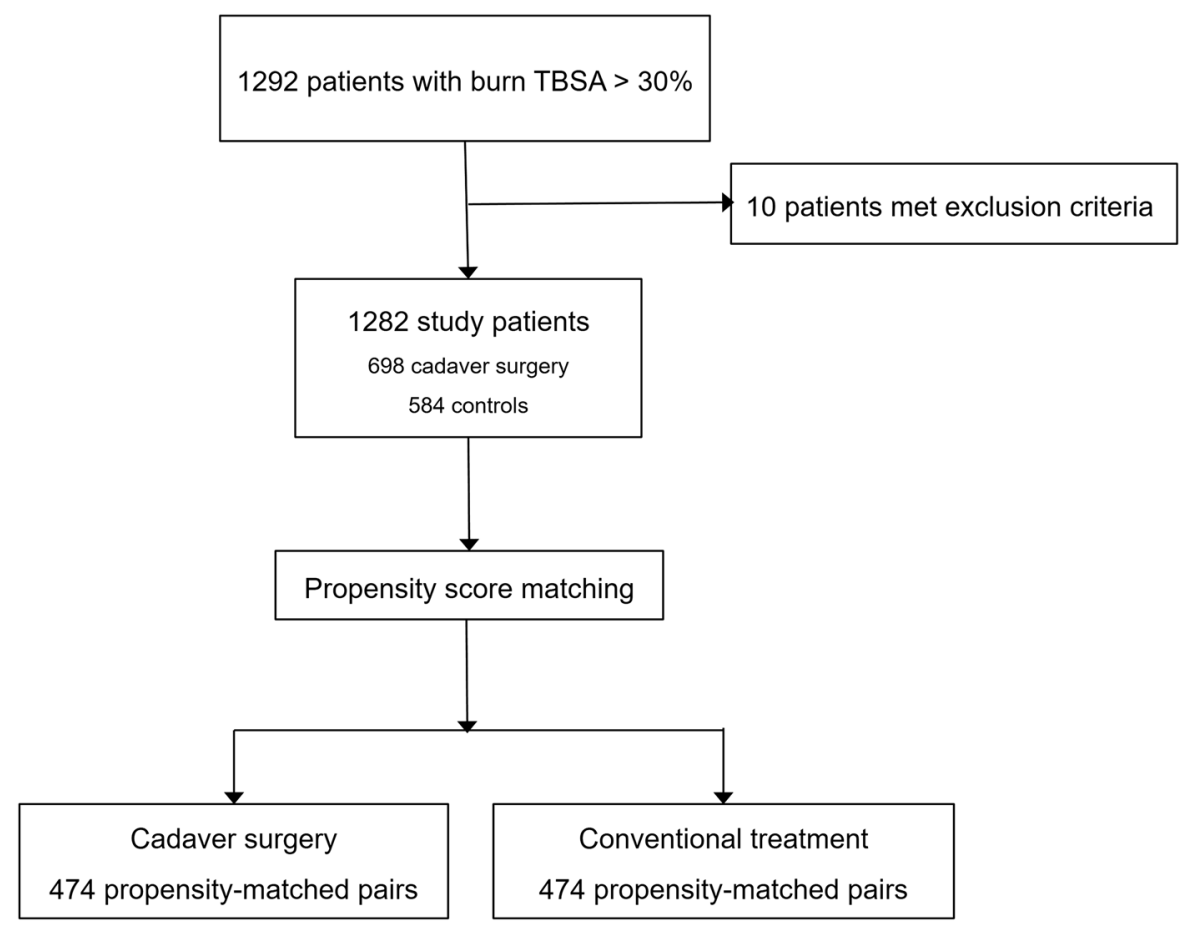

in a clinical trial that is commonly used in an observational study.

We performed logistic regression analysis to examine the association between cadaver skin allograft and 90-day in-hospital mortality. Survival curves were generated using the Kaplan-Meier method and compared using the log-rank test. We used Cox regression analysis to assess differences in in-hospital survival rates between patients with and without cadaver skin allograft in the propensity score-matched groups. A $p$ value $<0.05$ was considered statistically significant in all analyses. We performed all statistical analyses using IBM SPSS, version 19 (IBM Corp., Armonk, New York).

\section{Results}

We identified 1282 patients with burns affecting over $30 \%$ of TBSA treated at four hospitals located in four different cities (Seoul, Bucheon, Daejeon, and Busan) during the 9-year study period (Fig. 1). Of the 1282 patients, 488 were located in Seoul, 225 in Bucheon, 341 in Daejeon, and 228 in Busan. There were significant differences in age, sex, TBSA, and ABSI among patients at each hospital. The mortality rate in
Seoul, Bucheon, Daejeon, and Busan were 32.0, 29.3, 43.1 , and $38.6 \%$, respectively.

We categorized the patients according to whether they received cadaver skin allograft $(n=698)$ or not $(\mathrm{n}=584)$. In the cadaver group $51.0 \%(356 / 698)$ underwent cadaver skin allograft within 3 days of burn injury while $92.8 \%$ (648/698) underwent cadaver skin allograft within 7 days of burn injury. Furthermore, $10.1 \%(76 / 698)$ of patients in the cadaver group underwent cadaver skin allograft twice. Overall, $12.5 \%$ of the non-cadaver group was admitted to the general ward rather than the ICU. It is common for patients with more than $30 \%$ TBSA to be admitted to the ICU; however, if the burn surgeon decides that such patients have a large proportion of superficial wound area, they are admitted to the general ward. We corrected those imbalances and differences between the two groups through the propensity score-matching and generated 474 propensity score-matched pairs. Table 1 shows the general characteristics of the unmatched and propensity score-matched groups. In the unmatched groups, patients were more likely to have received cadaver skin allograft if they had fullthickness burns and were admitted to the ICU. After propensity score matching, baseline patient characteristics were well balanced between groups (Table 1). 
Table 1 General characteristics in the unmatched and propensity-matched groups

\begin{tabular}{|c|c|c|c|c|c|c|}
\hline \multirow[t]{2}{*}{ Variables } & \multicolumn{3}{|l|}{ Unmatched groups } & \multicolumn{3}{|l|}{ Matched groups } \\
\hline & Cadaver $(n=698)$ & Non-cadaver $(\mathrm{n}=584)$ & $p$ & Cadaver $(n=474)$ & Non-cadaver $(n=474)$ & $p$ \\
\hline Male (n) & $547(78.4)$ & $441(75.5)$ & 0.23 & $365(77.0)$ & $369(77.8)$ & 0.76 \\
\hline Age [y (SD)] & $47.4(16.5)$ & $48.9(17.9)$ & 0.12 & $48.6(17.5)$ & $49.4(16.2)$ & 0.44 \\
\hline TBSA [\% (SD)] & $51.8(18.2)$ & $50.7(23.9)$ & 0.37 & $54.2(24.8)$ & $52.4(18.9)$ & 0.16 \\
\hline Full thickness (n) & $628(90.0)$ & $424(72.6)$ & $<0.001$ & 407 (85.9) & $415(87.6)$ & 0.44 \\
\hline Inhalation (n) & $249(35.7)$ & $210(36.0)$ & 0.92 & $210(44.3)$ & $198(41.8)$ & 0.43 \\
\hline ICU admission (n) & $672(96.3)$ & $511(87.5)$ & $<0.001$ & $451(95.1)$ & $455(96.0)$ & 0.53 \\
\hline Use of ventilator (n) & $237(34.0)$ & $203(34.8)$ & 0.76 & $195(41.1)$ & $196(41.4)$ & 0.95 \\
\hline ABSI (SD) & $9.9(2.3)$ & $9.7(3.1)$ & 0.73 & $10.2(3.1)$ & $10.2(2.5)$ & 0.84 \\
\hline
\end{tabular}

$S D$ standard deviation, TBSA total body surface area, $I C U$ intensive care unit, $A B S I$ abbreviated burn severity index

Overall 90-day in-hospital mortality rate among all patients was $35.3 \%$ (453/1282). There was a significant difference in 90-day in-hospital mortality between the two groups for both unmatched (cadaver vs. non-cadaver, 31.7 vs. $39.7 \%$; difference, $8.0 ; 95 \%$ CI $2.8-13.3$ ) and propensity-matched groups (37.8 vs. 47.3\%; difference, 9.5; 95\% CI 3.2-15.8; Table 2). Logistic regression analyses showed a significant association between receiving a cadaver skin allograft and lower 90-day in-hospital mortality in the propensity-matched groups (odds ratio, 0.42; 95\% CI 0.29-0.62). The survival curve is presented in Fig. 2. The 90-day in-hospital mortality rate was lower in the cadaver group than that in the non-cadaver group (37.8 vs. $47.3 \% ; p<0.001$ by log-rank test). Cox regression analysis showed a significant difference in 90-day inhospital mortality between cadaver and non-cadaver groups in the propensity-matched groups (hazard ratio, 0.39; 95\% CI 0.33-0.49).

\section{Discussion}

It has been approximately 30 years since our institutions began to treat patients with burns. The mortality rate of patients with major burns in our institutions has gradually decreased as surgical techniques and multidisciplinary teamwork have advanced. The use of cadaver skin allograft is the most common and important surgical approach in treating patients with major burns in our institutions. Herein, we analyzed the impact of cadaver skin allograft on the mortality rate of patients with major burns.

Cadaver skin is one of the skin substitutes applied to patients with major burns for temporary skin coverage.

Table 2 Comparison of 30-day, 60-day, and 90-day in-hospital mortality rates

\begin{tabular}{|c|c|c|c|c|c|}
\hline Groups & \multicolumn{2}{|c|}{ Cadaver $(\%)$} & \multicolumn{2}{|c|}{ Non-cadaver $(\%)$} & Difference ( $95 \%$ conference interval) \\
\hline \multicolumn{6}{|l|}{ 30-Day in-hospital mortality } \\
\hline Unmatched groups & 25.6 & $(179 / 698)$ & 38.4 & $(224 / 584)$ & $12.7 \%(7.6-17.8)$ \\
\hline Propensity-matched groups & 31.2 & $(148 / 474)$ & 45.6 & $(216 / 474)$ & $14.3 \%(8.2-20.5)$ \\
\hline \multicolumn{6}{|l|}{ 60-Day in-hospital mortality } \\
\hline Unmatched groups & 30.9 & $(216 / 698)$ & 39.6 & $(231 / 584)$ & $8.6 \%(3.4-13.9)$ \\
\hline Propensity-matched groups & 37.3 & $(177 / 474)$ & 47.0 & $(223 / 474)$ & $9.7 \%(3.4-16.0)$ \\
\hline \multicolumn{6}{|l|}{ 90-Day in-hospital mortality } \\
\hline Unmatched groups & 31.7 & $(221 / 698)$ & 39.7 & $(232 / 584)$ & $8.0 \%(2.8-13.3)$ \\
\hline Propensity-matched groups & 37.8 & $(179 / 474)$ & 47.3 & $(224 / 474)$ & $9.5 \%(3.2-15.8)$ \\
\hline
\end{tabular}


Fig. 2 Survival plot with severe burns [total body surface area

(TBSA) $>30 \%$ ] treated with (solid line) or without (dashed line) cadaver surgery in propensity scorematched groups. The 90 day in-hospital mortality rate was lower in the cadaver group than non-cadaver group (37.8 vs. $47.3 \%$; $p<0.001$ by log-rank test)

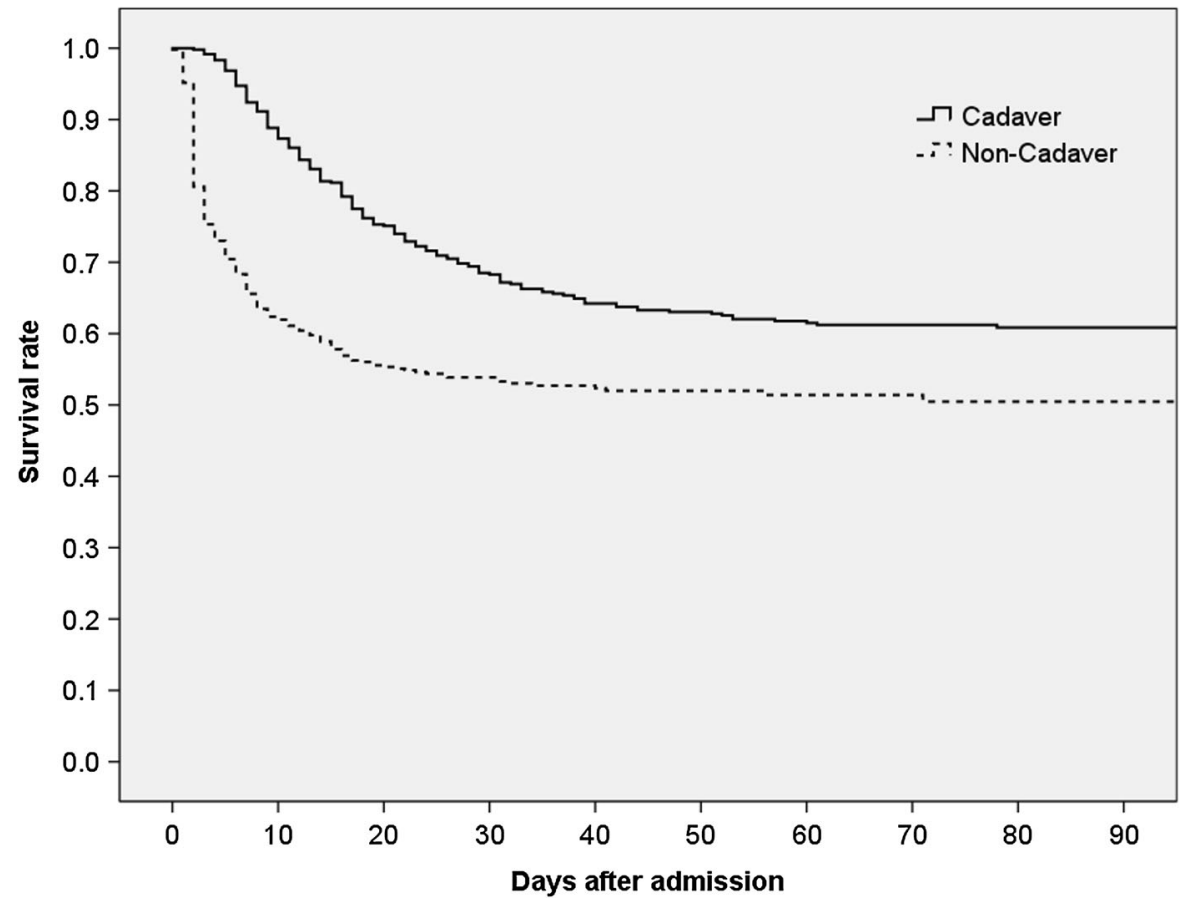

Skin substitutes for temporary wound covering are clinically useful in four settings in burn care: (1) as a dressing on donor sites to facilitate pain control and epithelialization; (2) as a dressing on clean superficial wounds, for the same reasons; (3) to provide temporary physiologic closure of deep dermal and fullthickness wounds after excision, while awaiting autografting or healing of underlying widely meshed autografts (wound bed preparation); and (4) as a 'test' graft in questionable wound beds (Sheridan and Tompkins 1999). Skin substitutes, specifically cadaver skin, can also be used in combination with widely expanded autografts in patients with large burns (sandwich grafting technique) (Khoo et al. 2010; Vloemans et al. 2002). Khoo et al. (2010) treated $67.4 \%$ (29/43) of patients with burns using cadaver skin for wound bed preparation, and Vloemans et al. (2002) reported using the sandwich grafting technique, overlaying over-widely meshed autograft, or expanded postage stamp autografting in 35\% (70/200) of patients studied. Traditionally, cadaver skin was rarely used at our institutions for the first and second setting and the sandwich grafting technique was not used because the Korean National Health Insurance had not approved it. Therefore, burn surgeons in our institution used the cadaver skin for the third and fourth setting.

Our burn centers have used cadaver skin since 2005. Since 2010, the Korean National Health Insurance Service began coverage of the use of cadaver skin in patients with burns of $\geq 30 \%$ of TBSA and cadaver skin has become the most costeffective material for temporary wound covering in Korea. In 2016, the common protocol of cadaver skin grafting at the four hospitals included in this study was reviewed on three occasions through discussions involving 24 burn surgeons working within the institutions.

We found that surgeons at our institutions had different views concerning the use of cadaver skin regarding the treatment of patients with $<40 \%$ of TBSA burned. Most surgeons at our institutions preferred cadaver skin allografting for patients with $>50 \%$ of TBSA burned. According to our results, the frequency of cadaver skin use was $50.0 \%$ in patients with burns affecting $30-49 \%$ of TBSA and $63.0 \%$ in patients with $>50 \%$ of TBSA burned. Fletcher et al. (2013) reported that $49.4 \%$ of patients with burns involving 30-49\% of TBSA were treated with cryopreserved allograft and $>90 \%$ of patients with burns 
involving $\geq 50 \%$ of TBSA were treated with cryopreserved allograft.

While our results are comparable with those reported by Fletcher et al. (2013) for patients with $30-49 \%$ of TBSA burned, there is a considerable difference in practice for patients with $>50 \%$ of TBSA burned. Two reasons may explain this discrepancy. First, there are differences in sample size between the two studies. The study by Fletcher et al. (2013) included approximately 50 patients, while ours included about 500. A larger sample size may have decreased the difference between the two groups. Second, Fletcher et al. (2013) included many patients (87\% of subjects) with burns and concomitant polytrauma, which may explain the more aggressive approach to cadaver skin allografting, while most patients in our study presented with isolated burns.

In studies by Blome-Eberwein et al. (2002) and Khoo et al. (2010), 85 and 29 patients with burns for which cadaver skin was applied had a 33 and $29 \%$ mortality rate, respectively. However, no control groups were included in the studies. In the current study, we had a control group that did not undergo cadaver skin allografting and compared the survival rates between the two groups. We found that the cadaver group had a higher survival rate than did the control group in the early phase of injury. According to Fig. 2, the difference in mortality rates between the cadaver and non-cadaver groups began to increase 2-3 days after burn injury, and there seemed to be no change 10-15 days after the burn injury. This may be related to the timing of the cadaver skin allograft. Overall, $51.0 \%(356 / 698)$ of the cadaver group underwent cadaver skin allograft within 3 days of the burn injury while $31.1 \%$ (217/698) underwent cadaver skin allograft within 2 days of the burn injury. Cadaver skin might have played a role in providing a physical barrier against bacteria, loss of protein, and fluid. Cadaver skin could not have had a direct impact on mortality approximately 15 days after the burn injury since it was removed by this time.

The difference between the two groups gradually decreased and then was maintained beginning at 60 days following the burn injury. This is because death occurred in the control group mainly in the early phase of injury, but rarely occurred at 60 days or more after the burn injury. Therefore, cadaver skin allograft in major burns is probably related to survival rate in the early phase of injury, decreasing the mortality rate by about half (odds ratio, 0.42 ).

This study has several limitations. First, this was a retrospective study. Therefore, although it included approximately 1300 patients with burns admitted to four hospitals, with approximately 700 patients undergoing cadaver skin allografting, bias might have existed due to the retrospective design of the study. We did not identify the reasons to explain why patients could not undergo cadaver skin allograft; therefore, we could not estimate the effect of these variables on mortality rate. In addition, we could not use the cadaver skin due to economic reasons and insurance policies. Therefore, the results should be interpreted with caution. A prospective study will be necessary to overcome these biases. Second, our data was retrospective and observational, without randomization. Even though we adopted propensity score matching to adjust for differences in baseline characteristics and disease severity, there may still have been bias in the form of confounders that were not measured. Pathogenic microorganisms and burn-wound sepsis can affect the prognosis. However, we did not include these factors in our initial research design process because of missing or incomplete data. However, we included the major factors that potentially affect mortality in patients with severe burns, such as age, sex, TBSA, full thickness, inhalation, use of mechanical ventilation, and the baseline characteristics of selected patients with severe burns were well balanced in the propensity score-matched groups. Although large randomized trials are necessary to confirm these results, it may be difficult to perform such trials in a large number of patients with this life-threatening condition. Third, the majority of burn surgeons in this study used cadaver skin for wound bed preparation. It was rare to use cadaver skin for biologic dressing or employ the sandwich technique with autograft. Fourth, our data did not include the proportion of fullthickness burns relative to the TBSA burned, although this affected patients' prognosis and the decision for early excision. Finally, this study evaluated the effect of cadaver skin on mortality rate but did not examine the merits of the use of cadaver skin (pain relief, protection from heat and water loss, and reduction in hypertrophic scarring). 


\section{Conclusion}

Patients with major burns who underwent cadaver skin allografting had a lower mortality rate than those who did not. Cadaver skin allograft may improve the survival of patients with major burns, especially in the early phase of injury.

Acknowledgements This work was supported by the Soonchunhyang University Research Fund.

\section{Compliance with ethical standards}

Conflict of interest The authors declare that there are no conflict of interest.

\section{References}

Blome-Eberwein S, Jester A, Kuentscher M, Raff T, Germann G, Pelzer M (2002) Clinical practice of glycerol preserved allograft skin coverage. Burns 28:10-12

Burke JF, Bondoc CC, Quinby WC (1974) Primary burn excision and immediate grafting: a method shortening illness. J Trauma Acute Care Surg 14:389-395

Cope O, Langohr JL, Moore FD, Webster RC Jr (1947) Expeditious care of full-thickness burn wounds by surgical excision and grafting. Ann Surg 125:1

Dhennin C, Desbois I, Yassine A, Benissad H, Lignee J (2002) Utilisation of glycerolised skin allografts in severe burns. Burns 28:21-25

Druecke D, Steinstraesser L, Homann H, Steinau H, Vogt P (2002) Current indications for glycerol-preserved allografts in the treatment of burn injuries. Burns 28:26-30

Engrav LH, Heimbach DM, Reus JL, Harnar TJ, Marvin JA (1983) Early excision and grafting vs. nonoperative treatment of burns of indeterminant depth: a randomized prospective study. J Trauma 23:1001-1004
Fletcher JL, Caterson E, Hale RG, Cancio LC, Renz EM, Chan RK (2013) Characterization of skin allograft use in thermal injury. J Burn Care Res 34:168-175

Greenfield LJ, Mulholland MW, Oldham KT, Zelenock GB, Lillemoe KD (2001) Surgery: scientific principles and practice. Lippincott Williams \& Wilkins, Philadelphia

Herndon DN, Barrow RE, Rutan RL, Rutan TC, Desai MH, Abston S (1989) A comparison of conservative versus early excision. Therapies in severely burned patients. Ann Surg 209:547

Janžekovic Z (1970) A new concept in the early excision and immediate grafting of burns. J Trauma Acute Care Surg 10:1103-1108

Khoo T, Halim A, Saad AM, Dorai A (2010) The application of glycerol-preserved skin allograft in the treatment of burn injuries: an analysis based on indications. Burns 36:897-904

Lawton G, Dheansa B (2008) The management of major burns-a surgical perspective. Curr Anaesth Crit Care 19:275-281

Leon-Villapalos J, Eldardiri M, Dziewulski P (2010) The use of human deceased donor skin allograft in burn care. Cell Tissue Bank 11:99-104

Naoum JJ, Roehl KR, Wolf SE, Herndon DN (2004) The use of homograft compared to topical antimicrobial therapy in the treatment of second-degree burns of more than $40 \%$ total body surface area. Burns 30:548-551

Sheridan RL, Tompkins RG (1999) Skin substitutes in burns. Burns 25:97-103

Subcommittee S, Subcommittee A, Committee IPG (2016) ISBI practice guidelines for burn care. Burns 42:953-1021

Tompkins RG, Remensnyder JP, Burke JF, Tompkins DM, Hilton JF, Schoenfeld DA, Behringer GE, Bondoc CC, Briggs SE, Quinby W Jr (1988) Significant reductions in mortality for children with burn injuries through the use of prompt eschar excision. Ann Surg 208:577

Vloemans A, Schreinemachers M, Middelkoop E, Kreis R (2002) The use of glycerol-preserved allografts in the Beverwijk Burn Centre: a retrospective study. Burns 28:2-9 